\title{
Morfometria do coração e dos vasos da base e sua implicação no mergulho em Chelonia mydas ${ }^{1}$
}

\begin{abstract}
Janine K.F.S. Braz ${ }^{2 *}$, Maria Sara M. Queiroz ${ }^{3}$, Moacir F. Oliveira ${ }^{4}$ e Carlos E.B. Moura ${ }^{3}$
ABSTRACT.- Braz J.K.F.S., Queiroz M.S.M., Oliveira M.F. \& Moura C.E.B. 2013. [Heart and base vessels morphometric and your implications in dive of Chelonia mydas.] Morfometria do coração e dos vasos da base e sua implicação no mergulho em Chelonia mydas. Pesquisa Veterinária Brasileira 33(Supl.1):32-38. Laboratório Multidisciplinar, Faculdade de Ciências da Saúde do Trairí, Universidade Federal do Rio Grande do Norte, Rua Trairi s/n, Centro, Santa Cruz, RN 59200-000, Brazil.E-mail: jani_karla@yahoo.com.br

This study aimed to characterize the morphology of the heart chambers, pulmonary arteries and aortas of Chelonia mydas. Eleven specimens were evaluated from $\operatorname{dead} C$. mydas collected on the coast of the state of Rio Grande do Norte, Brazil. The animals were necropsied to obtain heart, aorta and fragments of the right and left pulmonary vessels. The vessels were fixed in formalin and subjected to histological processing and staining by a modified Verhoff technique. The parameters width, height and base-apex ventricular circumference of the heart were measured with a caliper. In the species the microscopy of pulmonary arteries and aorta varied according to the antimere. The largest relative thickness of Cavum venosum (CV) assists the cardiac output during diving, and its smaller thickness is advantageous for the right ventricular dilation during deep immersion, while the quantification of elastic and muscle fibers of the tunica media of aortas and right and left pulmonary arteries showed that the medial layer of aorta dominates the elastic vs. muscle fibers; however the elastic fibers are not dominating in the pulmonary arteries. The angioarchitecture may be related to the ability to swim, favoring better use of oxygenated blood previously stored during the period of apnea.
\end{abstract}

INDEX TERMS: Anatomy, cardiovascular, green turtle, Chelonia mydas, histology.

RESUMO.- Objetivou-se caracterizar a morfologia das câmaras cardíacas e das artérias aortas e pulmonares da espécie Chelonia mydas. Foram avaliados 11 espécimes de $C$. mydas mortas coletadas no litoral do estado do Rio Grande do Norte, Brasil. Os animais foram necropsiados para a obtenção do coração, fragmentos das artérias aorta e pulmonares direita e esquerda. Os vasos adquiridos foram fixados em formol e submetidos ao processamento histológico de rotina e coloração com Técnica de Verhoff modificada.

\footnotetext{
${ }^{1}$ Recebido em 14 de setembro de 2013.

Aceito para publicação em 22 de novembro de 2013.

${ }^{2}$ Laboratório Multidisciplinar, Faculdade de Ciências da Saúde do Trairí (Facisa), Universidade Federal do Rio Grande do Norte (UFRN), Rua Trairi s/n Centro, Santa Cruz, RN 59200-000, Brasil. *Autor para correspondência: jani_karla@yahoo.com.br

${ }^{3}$ Departamento de Morfologia, Centro de Biociências, UFRGN, Cx. Postal 1524, Campus Universitário Lagoa Nova, Natal, RN 59072-970, Brasil.

${ }^{4}$ Departamento de Ciência Animal, Universidade Federal Rural do Semi-Árido, Av. Francisco Mota 572, Costa e Silva, Mossoró, RN 59625-900, Brasil.
}

Enquanto, do coração, os parâmetros largura, altura base-ápice e a circunferência ventricular foram mensurados por meio do paquímetro. Nessa espécie a microscopia das artérias pulmonares e artérias aortas variaram de acordo com o antímero. A maior espessura relativa do Cavum Venosum (CV) auxilia no bombeamento cardíaco durante o mergulho e sua menor espessura direita é uma vantagem para a dilatação ventricular durante a imersão profunda enquanto que a quantificação das lâminas elásticas e fibras musculares da túnica média das artérias aortas e pulmonares direita e esquerda comprovaram que a túnica média das aortas predomina o componente elástico vs. muscular, entretanto, nas artérias pulmonares o componente elástico não-predomina. Essa angioarquitetura pode estar relacionada com a capacidade de mergulho, favorecendo um maior aproveitamento do sangue oxigenado armazenado previamente durante o período de apneia.

TERMOS DE INDEXAÇÃO: Anatomia, cardiovascular, tartaruga-verde, Chelonia mydas, histologia. 


\section{INTRODUÇÃO}

As tartarugas marinhas atuais formam um grupo monofilético pertencente à Ordem Chelonia e à Subordem Cryptodira. As sete espécies apresentam-se distribuídas em duas famílias: Dermochelyidae e Cheloniidae. Todavia, na costa brasileira apenas cinco delas são encontradas: a tartaruga-verde (Chelonia mydas), tartaruga-de-pente (Eretmochelys imbricata), tartaruga-oliva (Lepidochelys olivacea), tartaruga-cabeçuda (Caretta caretta) e tartaruga-de-couro (Dermochelys coriacea) (Meylan \& Meylan 2000).

As tartarugas-verdes são classificadas pela International Union for Conservation of Nature (IUCN 2008) como vulneráveis a extinção, que direcionou as pesquisas a trabalhos sobre estratégias conservacionistas. Essa espécie, assim como as outras, apresenta adaptações morfológicas derivadas para uma vida no ambiente marinho, dentre elas os membros em formato de remo, com perda das articulações móveis nas falanges distais e variação de 3-4 dígitos em cada nadadeira (Meylan \& Meylan 2000). A morfologia externa composta por placas córneas e ósseas facilita o seu deslocamento na água, da mesma forma que as protege de predadores e aumenta sua hidrodinâmica (Silva \& Rodrigues 2009).

O coração das tartarugas, lagartos e cobras são formados de três câmaras: dois átrios e um ventrículo. 0 ventrículo é divido em três subcâmaras: o cavum pulmonale câmara mais ventral e se estende cranialmente ao óstio da artéria pulmonar; cavum arteriosum e cavum venosum situados dorsalmente são câmaras conectadas pelo canal interventricular (Murray 2006). As câmaras cardíacas são basicamente formadas pelo miocárdio com a presença rudimentar de músculos papilares e 2 projeções que emergem do septo interatrial que remetem a válvula mitral. As câmaras cardíacas apresentam variação na espessura muscular (Solomon et al. 1999). De acordo com Ashley (1955) a irrigação do miocárdio seria responsabilidade das artérias coronárias de pequeno calibre que se originam, possivelmente, do tronco braquiocefálico. A partir dos estudos feitos em Chelonia mydas por Quesada \& Madriz (1986), foram elaborados cinco arranjos de irrigação dos corações de acordo com o comportamento das artérias coronárias direita e esquerda, sua origem anatômica e distribuição.

As duas grandes aortas (a aorta direita e a aorta esquerda) e um tronco pulmonar que é divido em artéria pulmonar direita e esquerda (Wyneken 2001) estão localizados na base do coração. A aorta direita apresenta um ramo que compõe o tronco braquiocefálico que se continua mais abaixo para encontrar a aorta esquerda e bifurca-se originando a artéria tireoidiana e a artéria subclávia direita e esquerda. Já aorta esquerda irá se ramificar dorsolateralmente formando a artéria gástrica, a artéria celíaca e a artéria mesentérica cranial (Wykenen 2001). Não foram encontrados trabalhos sobre a descrição histológica de artérias aortas e pulmonares para essa espécie, apesar desse conhecimento fornecer subsídios importantes na elaboração de outros trabalhos através da análise comparativa entre as espécies marinhas da costa brasileira.

De acordo com Rice et. al. (2008) C. mydas caracteriza três tipos de mergulho em detrimento da profundidade e do período do dia: (1) o mergulho diurno pode exceder $120 \mathrm{~m}$ de profundidade em habitat natural, mas não é continuo durante todo o dia; (2) podem ocorrer mergulhos de pequena profundidade $(1,5-4 \mathrm{~m})$ com duração de 3 a 18 min; (3) já o mergulho noturno é caracterizado pelo descanso no fundo do mar, a uma profundidade igual ou superior a $20 \mathrm{~m}$. A tartaruga-de-couro, Dermochelys coriacea realiza mergulhos de $250 \mathrm{~m}$ de profundidade com duração de 10-20 min. Essa capacidade de mergulho é justificada pelas adaptações fisiológicas cardiovasculares e respiratórias a profundidades, mergulhos prolongados e o aumento da capacidade de reserva de oxigênio no sangue e nos tecidos (Lutcavage et al. 1992).

Apesar da presença de pulmões desenvolvidos e a respiração aérea, as tartarugas podem permanecer algumas horas embaixo da água, em apneia (Silva \& Rodrigues 2009). Durante o mergulho, a resistência pulmonar e a pressão são elevadas, dessa forma a circulação pulmonar passa a ser bipassada, fazendo com que a maior parte do sangue participe da circulação sistêmica. Essa habilidade poupa o coração fazendo-o fornecer oxigênio suficiente para manter a vascularização sistêmica, sendo essa uma vantagem sobre o mergulho de aves e mamíferos (Murray 2006). Esses mecanismos compensatórios, provavelmente devem influenciar a morfologia das câmaras cardíacas e estrutura das artérias pulmonares e aórticas.

As pesquisas com tartarugas marinhas são direcionadas para busca de estratégias conservacionista tornando os trabalhos no campo da fisiologia e morfologia cardiovascular restritos a condição de armazenamento de oxigênio e funcionamento dos pulmões durante o mergulho, a frequência cardíaca, fluxo sanguíneo cardíaco, comportamento dos pulmões durante o período imerso em água e estudos de hemogramas (Southwood et al. 1999, Hay et al. 2000, Wyneken 2001, Lopes-Mendilaharsu et al. 2008, Santos et al. 2009). 0 que torna esse trabalho uma ferramenta auxiliar no campo da investigação dos padrões filogenéticos cardiovasculares existentes na ordem permitindo estabelecer, e, dessa forma, gerar contribuições significantes para compreensão da anatomia circulatória das espécies. Nesse caso, é de grande valor reafirmar que não existem informações detalhadas acerca da histologia do sistema cardiovascular de tartarugas marinhas para a maioria das estruturas. Além disso, não há uma análise mais aprofundada do volume cardíaco em detrimento da atividade de mergulho. Com isso, o presente trabalho tem por objetivo avaliar a morfometria das câmaras e microscopia das artérias pulmonares e aórticas e sua possível implicação no mergulho dessa espécie.

\section{MATERIAL E MÉTODOS}

Foram utilizadas tartarugas marinhas da espécie Chelonia mydas encontradas mortas na costa litorânea do estado do Rio Grande do Norte - Brasil ou que foram a óbito durante o processo de reabilitação no Aquário Natal no período de novembro de 2009 a setembro de 2011, totalizando 11 animais sob a autorização do TAMAR/ICMBio (Licença no 21647-1 IBAMA/RN).

Biometria dos animais. 0 comprimento curvilíneo da carapaça (CCC) foi mensurado a partir do ponto médio anterior 
do escudo nucal ao extremo das placas supracaudares e largura curvilínea da carapaça (LCC) (Bolten 2000, Work 2000). Essas informações serviram como base para a identificação da faixa de tamanho em que cada espécime se encontrava de acordo com Hirth (1997). Tornando possível afirmar que todos os espécimes coletados estavam na fase juvenil (Quadro 1).

Quadro 1. Médias do Comprimento Curvilíneo da Carapaça (CCC) e Largura Curvilínea da Carapaça (LCC) das espécies Chelonia myda. Natal, 2011

\begin{tabular}{cccc}
\hline Espécime & Faixa etária & CCC $(\mathrm{cm})$ & LCC $(\mathrm{cm})$ \\
\hline 172/02 & Juvenil & 31 & 28 \\
CM06 & & 28 & 25 \\
CM07 & & 29 & 26 \\
CM08 & & 37 & 33 \\
CM09 & 35 & 30 \\
CM10 & 51 & 46 \\
CM11 & 60 & 59 \\
CM12 & 38 & 35 \\
CM13 & 34 & 33.2 \\
CM14 & 41 & 39 \\
CM15 & 34 & 30 \\
& & $38,00 \pm 9,21 \mathrm{~cm}$ & $34,93 \pm 9,21 \mathrm{~cm}$
\end{tabular}

Análise da morfometria do coração. Após a remoção do coração da cavidade pericárdica ainda com o órgão fechado e em posição anatômica (Fig.1A) foram obtidas as medidas da altura do ventrículo sentido base-ápice (Fig.1C), largura (Fig.1B), circunferência (Fig.1D), espessura da câmara direita (Cavum Venosum) e da câmara esquerda (Cavum Arteriosum) do coração da Chelonia mydas com o uso de paquímetro (Fig.1E,F). Após a abertura do coração foram mensuradas em vista dorsal e ventral, por meio de um paquímetro, a espessura da câmara direita (Cavum Venosum) e da câmara esquerda (Cavum Arteriosum) (Fig.2).

Análise e processamento histológico das artérias. Foram coletados fragmentos de $1 \mathrm{~cm}$ das artérias aórticas e artérias pulmonares direita e esquerda de 8 tartarugas descongeladas e uma a fresco. Em virtude das condições do coração após o descongelamento, alguns espécimes foram desconsiderados para essa análise. Os fragmentos foram submetidos a fixação em solução de formol tamponado a $10 \%$ por 24 horas. Decorrido esse período foram aplicados os procedimentos histológicos (desidratação, diafanização e inclusão em parafina) e submetidas a coloração com Hematoxilina e Eosina seguindo a metodologia proposta por Maia (1979) para análise preliminar dos cortes. As lâminas permanentes confeccionadas foram observadas ao microscópio de luz Olympus BX41, e microfotogradas para análises e comparações com câmera digital Nikon DXM1200, ambos com suporte acoplado ao microcomputador. As imagens foram capturadas com auxílio do software Act-1.

Análise em Microscopia de Varredura (MEV). Foram confeccionadas lâminas com cortes de $10 \mu \mathrm{m}$, seguindo protocolo de histologia proposto por Maia (1979). As lâminas não receberam coloração, sendo apenas submetidas ao xilol para retirar o excesso de parafina, após a secagem sofreram metalização para visualização na MEV. As fotomicrografias foram obtidas do Microscópio Eletrônico de Varredura (MEV), XL-30 - ESEM (Phillips) do Núcleo de Estudos de Petróleo e Gás da UFRN.

Análise quantitativa das lâminas elásticas e musculares da túnica média das artérias aortas e pulmonares. As lâminas confeccionadas das artérias aorta (direita e esquerda) bem como, das artérias pulmonares (direita e esquerda) foram coradas como a técnica de Verhoeff modificada, a saber que em preto são coradas as lâminas elásticas pela hematoxilina férrica de Verhoeff, e contracorados com eosina. As lâminas foram observadas ao microscópio de luz Olympus BX41, e microfotografadas no formato (tiff.) e resolução 1280 x 1024 conforme descrito na análise histológica. Foram obtidos 4 campos por lâmina com auxílio do software Act-1 e analisadas no Software ImageProPlus. A quantidade de lâminas elásticas foi determinada pelo quociente entre área ocupada por estas, sobre a área total do campo. 0 mesmo foi feito para fibras musculares. Os resultados foram expressos em porcentagem média.

Análise estatística. A análise de variância de Kruskal-Wallis foi utilizada para comparar as porcentagens médias de fibras elásticas e musculares nas artérias aortas e pulmonares direitas e esquerdas. Foi aplicado o teste não-paramétrico de Mann-Whitney para duas amostras independentes, para comparar a espessura média do Cavum arteriosum (C.A.); Cavum venosum (C.V.). Todas as análises foram realizadas pelo software Graph Prism 4.0.

\section{RESULTADOS}

\section{Morfologia do coração e vasos da base}

O coração da tartaruga é envolvido por um saco fibroseroso, pericárdio, localizado próximo ao acrômio e o processo coracóide da escápula; ventralmente, aos pulmões e lateralmente aos lobos do fígado. 0 formato anatômico é piramidal com ápice voltado para baixo e identificado pelo gubernáculo, caudoventralmente. 0 coração dos espécimes analisados possui 3 cavidades, sendo 2 átrios e 1 ventrículo. 0 ventrículo por sua vez é subdividido em Cavum vensosum, a câmara direita ventricular, e o Cavum arteriosum a câmara esquerda ventricular. Cavum pulmonale fica entre as duas câmaras com maior proximidade com Cavum arteriosum.

Os animais possuem três grandes vasos: a aorta esquer$\mathrm{da}$, aorta direita e o tronco pulmonar. A base das aortas possuem o septo inter-aórtico formado por cartilagem. Nessa espécie a presença de bulbo aórtico não foi verificada. As artérias pulmonares estavam presentes na bifurcação do tronco pulmonar, na porção cranial do ventrículo. Os resultados demonstraram que o Cavum arteriosum possui maior espessura da parede marginal quando comparado à parede do Cavum venosum (Quadro 2).

\section{Microscopia das artérias aortas e pulmonares}

Microscopicamente, as artérias aorta e pulmonares apresentaram a parede vascular contendo as três túnicas histológicas (Fig.3). A parede arterial possuía as túnicas: íntima (mais interna), média e adventícia (mais externa). Na microscopia eletrônica de varredura foi observada a disposição das fibras em cada camada dos vasos em que está visível o endotélio da túnica íntima, seguido da túnica média com fibras musculares, mais delgadas, e lâminas elásticas mais espessas e a túnica adventícia com tecido conjuntivo (Fig.4).

A análise dos resultados de quantificação das lâminas elásticas e fibras musculares da túnica média das artérias aortas e pulmonares direita e esquerda comprovaram que na túnica média das aortas predomina o componente elástico vs. muscular, entretanto, nas artérias pulmonares o componente elástico não-predomina. (Quadro 3).

\section{DISCUSSÃO}

O sistema circulatório é o que sofre maior variação quando comparado aos outros órgãos e sistemas, isso pode desen- 
cadear variações adaptativas que podem ser sutis ou drásticas dependendo da espécie (Franklin \& Axelsson 1994, Wyneken 2001). As adaptações fisiológicas cardiovasculares e respiratórias podem ser ocasionadas para suportar mergulhos prolongados gerando aumento da capacidade de reserva de oxigênio no sangue e nos tecidos, como foi observado para a espécie Tartarugas-de-couro (Dermochelys coriacea), cujo mergulho alcança 250 metros e dura de 10-20 minutos (Lutcavage et al. 1992). A tartaruga-verde é caracterizada em três tipos de mergulho em detrimento da profundidade de 30 metros ao longo do dia (Rice \& Balazs 2008). Contudo, não há estudos que possam inferir a ca-
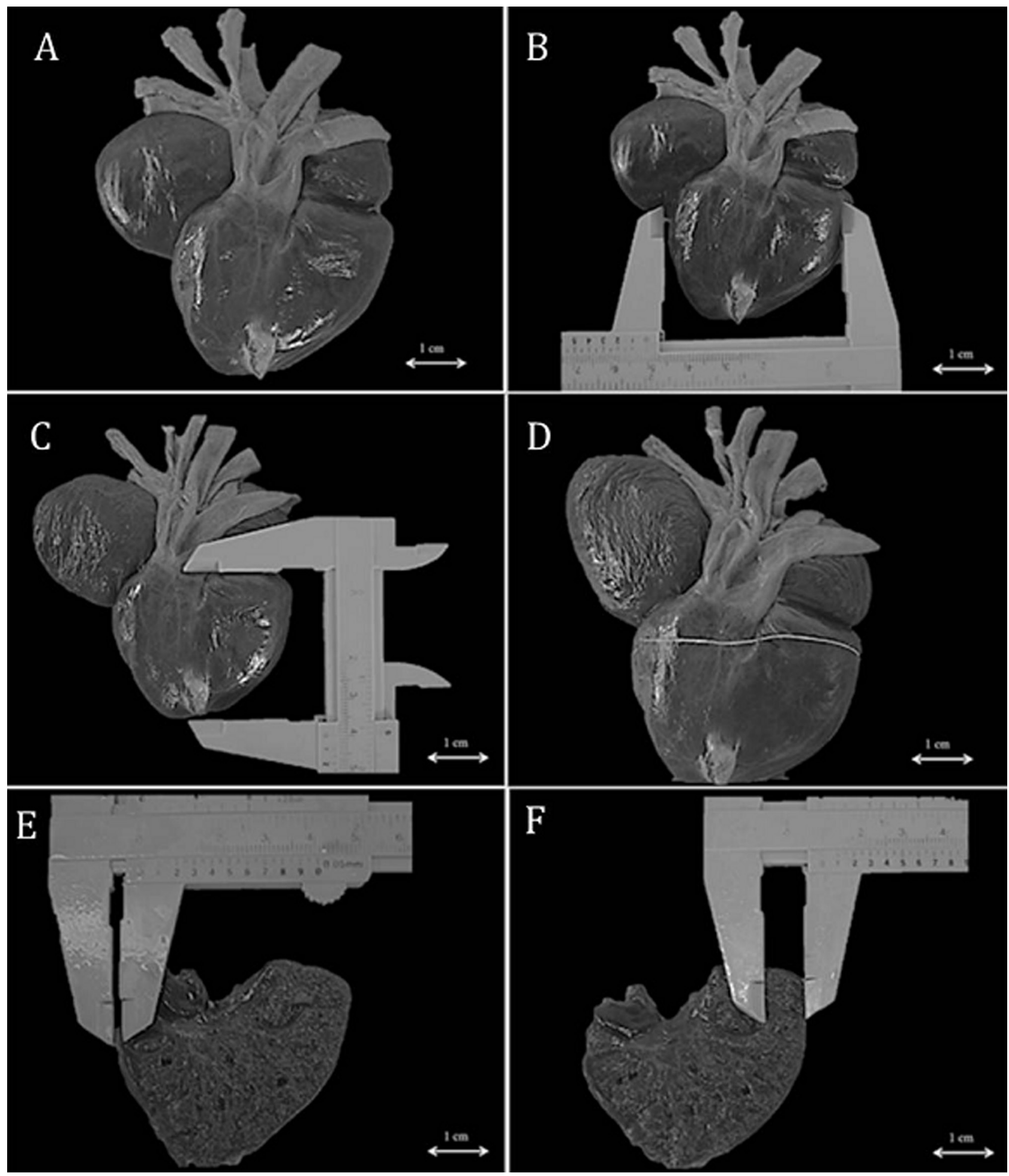

Fig.1. Biometria ventricular interna e externa: (A) Vista ventral do coração, (B) Medida da largura, (C) Medida altura base-ápice, (D) Medida da circunferência, (E) Vista dorsal-interior, espessura da câmara direita,; (F) Vista dorsal-interior, espessura da câmara esquerda. 


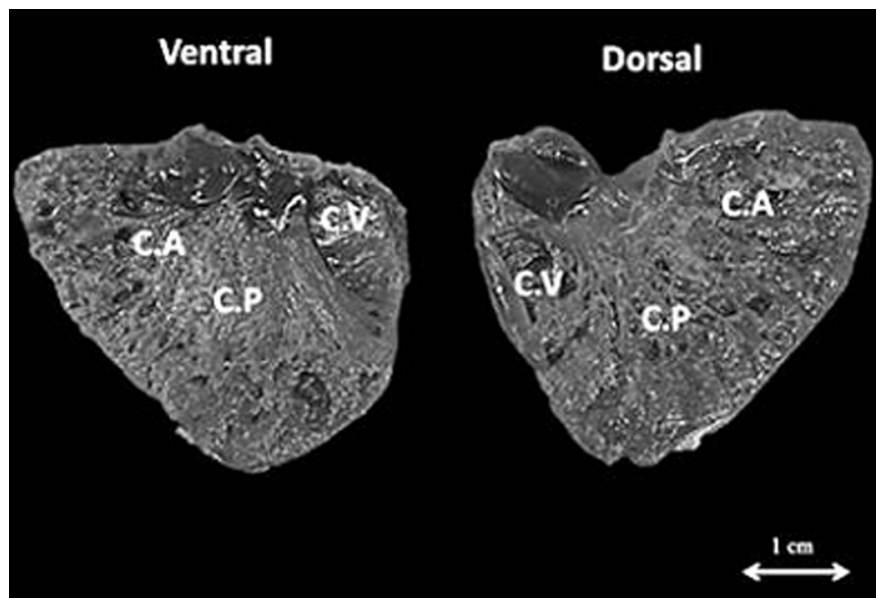

Fig.2. Câmaras no interior do ventrículo de Chelonia myda: Cavum arteriosum (C.A.), Cavum pulmonale (C.P.), Cavum venosum (C.V.).

Quadro 2. Espessura média das cavidades Cavum arteriosum (C.A.) e Cavum venosum (C.V.) ventricular do coração em Chelonia mydas. Natal, 2011

\begin{tabular}{|c|c|c|c|c|}
\hline \multicolumn{2}{|c|}{ Cavum venosum $(\mathrm{cm})$} & \multirow[t]{2}{*}{ Razão* (CV/CA) } & \multicolumn{2}{|c|}{ Cavum arteriosum $(\mathrm{cm})$} \\
\hline & & & & \\
\hline $0,23 \pm 0,15^{b}$ & $0,22 \pm 0,09^{\mathrm{B}}$ & $1,1: 2$ & $0,82 \pm 0,56^{a}$ & $0,80 \pm 0$ \\
\hline
\end{tabular}

$\overline{\mathrm{a}, \mathrm{b}}$ Indicam diferença estatística na espessura das cavidades na vista ventral $(\mathrm{p}<0,05) ; \mathrm{A}, \mathrm{B}$ Indicam diferença estatística na espessura das cavidades na vista dorsal $(\mathrm{p}<0,05) ;{ }^{*}$ Razão = Média(C.V. Ventral ou dorsal) / Média(C.A. Ventral ou dorsal) x 10.

pacidade de mergulho peculiar da espécie como um fator influenciador para o surgimento de adaptações fisiológicas cardiovasculares.

É notório que as adaptações começam pelo coração. A câmara ventricular direita em vertebrados como peixes, anfíbios, serpentes, crocodilos, cetáceos e mamíferos terrestres carnívoros é de parede delgada, já a esquerda é caracterizada por uma espessa parede esponjosa e compacta para peixes, anfíbio e répteis (tartarugas) (Davis 1964, Franklin \& Axelsson 1994, Solomon et al. 1999). Dessa forma o ventrículo possui adaptações morfológicas à circulação, como a espessura maior do ventrículo esquerdo

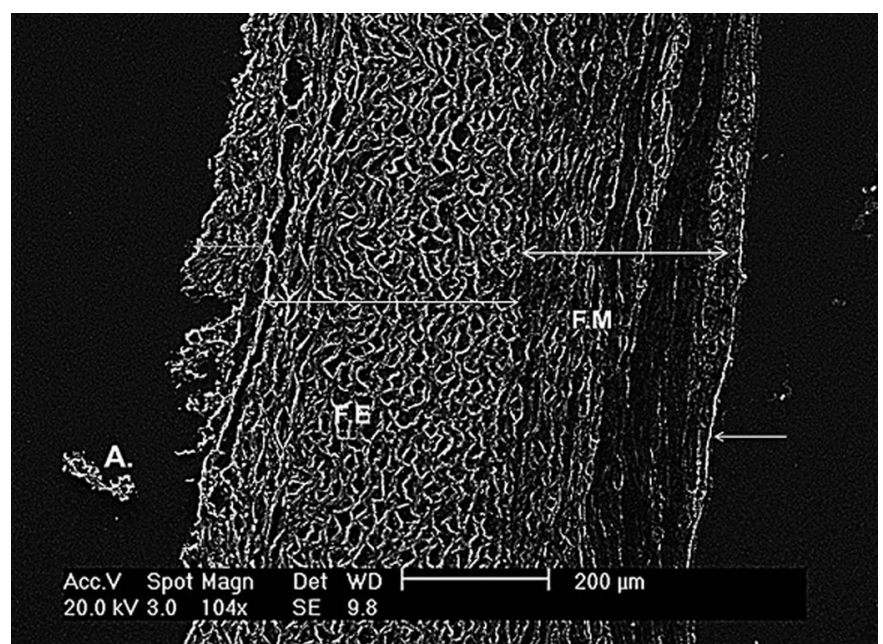

Fig.4. Aorta direita da tartaruga 172/02. As setas indicam as túnicas do vaso: Túnica adventícia $(\longleftrightarrow \rightarrow)$, Túnica média $(\longleftrightarrow \rightarrow)$ com fibras elásticas (F.E.), Túnica médica $(\leftarrow \rightarrow)$ com fibras musculares (F.M.); Endotélio $(\leftarrow)$, Artefato da lâmina (A.).

Quadro 3. Área média, em porcentagem, das lâminas elásticas e fibras musculares na túnica média das artérias aortas e pulmonares direita e esquerda em Chelonia mydas. Natal, 2011

\begin{tabular}{|c|c|c|c|c|c|c|}
\hline \multicolumn{3}{|c|}{ Lâminas elásticas(\%) } & \multicolumn{4}{|c|}{ Fibras musculares (\%) } \\
\hline Aorta & Puln & 101 & & orta & Pulm & onar \\
\hline Direita Es & Direita & $\mathrm{la}$ & Direita & Esquerda & Direita & Esq \\
\hline $66^{\mathrm{a}}$ & 51,90 & 7,23 & $0,23^{b}$ & $36,46^{\mathrm{B}}$ & 48,10 & 7 \\
\hline
\end{tabular}

$\overline{a, b}$ Indicam diferença altamente significativa $(p<0,01)$, ${ }^{A, B}$ indicam diferença significativa $(\mathrm{p}<0,05)$.

em mamíferos em relação ao ventrículo direito (Drabek \& Burns 2002).

Pesquisas com mamíferos aquáticos revelam que essa relação é menor, justificada por uma provável resistência dos pulmões em virtude do mergulho profundo e a parede ventricular direita delgada é uma vantagem à dilatação ventricular durante o mergulho em cetáceos (Bisaillon et al. 1987). Nesse estudo foi observado que C. mydas possui uma relação pequena entre o Cavum Venosum e Cavum Ar-
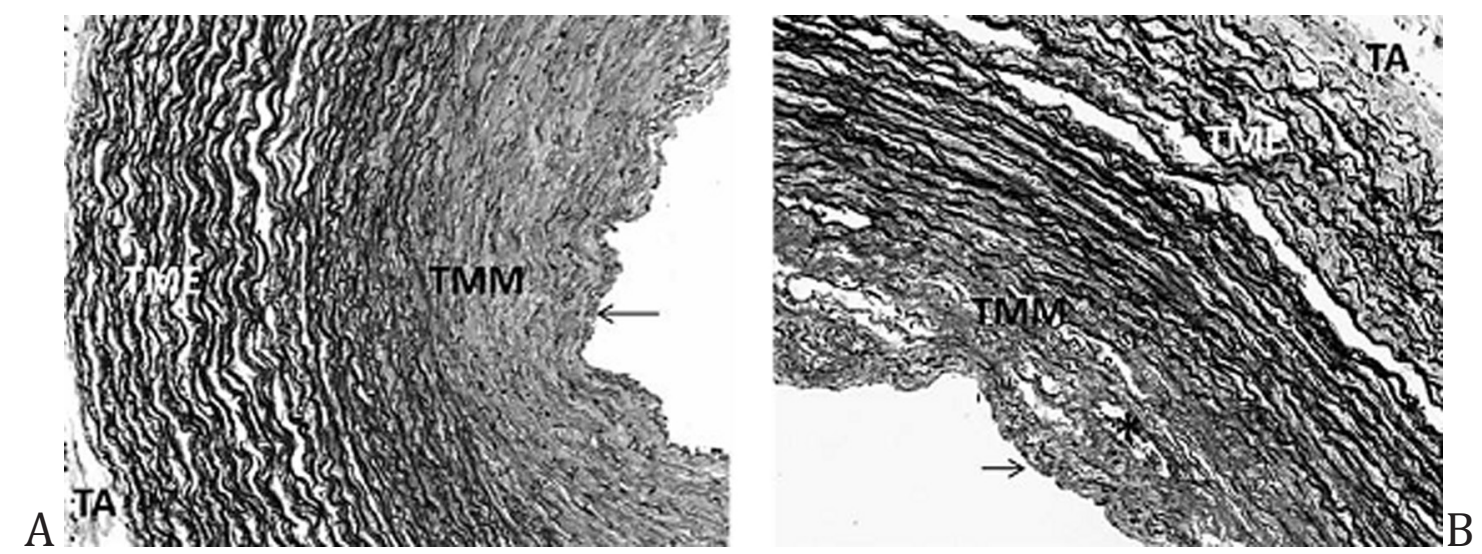

Fig.3. (A) Aorta Direita. (B) Artéria Pulmonar Direita. Endotélio $(\rightarrow)$.Túnica média com fibras musculares (TMM), Túnica médica com lâminas elásticas (TME), Túnica adventícia (TA), Capilares sanguíneos(*). Coloração de Verhoeff modificado. Aumento 100x. 
teriosum (1,1:2), indicativo de menor espessamento relativo da parede do Cavum Venosum, maior responsável pelo bombeamento do sangue para os pulmões nessa espécie, já que a sístole cardíaca em tartarugas ocorre de forma caudal-cranial, ao contrário do que é encontrado em mamíferos (Valete et al. 2008, Wyneken 2009).

Esse resultado de acordo com Bisaillon et al. (1987) pode inferir que a parede delgada da câmara ventricular direita é uma vantagem para a dilatação ventricular durante a imersão profunda, assim como foi encontrado em filhotes de leão-marinho-do-sul a delgada espessura da parede ventricular direita poderia constituir um mecanismo para impedir o colapso pulmonar do ventrículo em virtude da variação de pressão durante o mergulho (Junin \& Belerenian 1990).

Além disso, outra implicação para adaptação morfológica ao mergulho foi encontrada em pinípedes e cetáceos a dilatação na base da artéria aorta, bulbo aórtico, com grande quantidade de fibras elásticas, que pode auxiliar a manter a pressão arterial, bem como do fluxo sanguíneo nas artérias coronárias durante o mergulho (Drabek \& Burns 2002). Essa concentração de fibras elásticas na aorta ascendente serve, provavelmente, para manter a pressão sanguínea e consequentemente garantir a perfusão tecidual adequada mesmo durante o mergulho. Segundo Wyneken (2009) nas tartarugas não há evidências da existência de bulbo aórtico.

No entanto, no presente trabalho foi observado, que as aortas de Chelonia mydas possuíam uma base mais larga, e não uma dilatação do arco aórtico, quando comparada aos outros répteis, surgindo dorsalmente e a direita do tronco pulmonar. Essa morfologia pode desempenhar um papel semelhante ao bulbo aórtico de pinípedes e cetáceos. As análises histológicas desse estudo apontaram maior proporção de componente elástico corroborando com essa hipótese para as aortas direita e esquerda.

Dessa maneira as aortas se contraem e relaxam em uma velocidade muito baixa, quando comparada a mamíferos, tornando a troca gasosa a nível celular mais lenta. Durante o mergulho esse comportamento pode auxiliar na redução de oxigenação do sangue nos pulmões durante o mergulho profundo, consequentemente, aumenta tempo em apneia das tartarugas, sem prejudicar o fluxo sanguíneo e a oxigenação dos tecidos (Valente, et al. 2008).

Já a constituição histológica das artérias pulmonares parece estar bem relacionada a ontogênese e ao comportamento de cada espécie em resposta ao seu habitat, já que os humanos apresentam apenas artérias pulmonares musculares, assim como os esquilos. Já animais como a foca, os coelhos, raposas e cachorros apresentam uma porção de fibras elásticas e muscular bem evidente nas artérias pulmonares (Best \& Heath 1961).

Em Chelonia mydas os resultados estatísticos revelam que a quantidade do componente muscular e elástico na túnica média das artérias pulmonares não diferiu, dessa forma, não podem ser consideradas predominantemente musculares. Segundo Best \& Heath (1961) a espessura das artérias pulmonares em crocodilos e tartarugas é delgada e está adaptada a morfologia do coração o qual está susceptível a stress hemodinâmicos na circulação pulmonar.

A constituição histológica é justificada como uma forma de suportar a hipertensão pulmonar existente, em virtude do mergulho. A prevalência de lâminas elásticas nas artérias pulmonares permite o fluxo contínuo de sangue durante a sístole e mantêm a pressão durante a diástole (que tende a ser menor no ventrículo) em mamíferos (Junqueira \& Carneiro 2008, Kierszenbaum 2008).

Durante o mergulho a pressão intrapulmonar torna-se maior que a pressão intraventricular, e nas artérias pulmonares é elevada permitindo a vasoconstrição com direcionamento do sangue não oxigenado para a circulação sistêmica, sem promover deformações significativas nas artérias. Dessa forma, há um aumento da resistência vascular, permitindo que o fluxo sanguíneo seja redirecionado para as aortas, que apresentam baixa resistilidade e permitem a passagem do sangue sem alterar o fornecimento de oxigênio para as áreas sistêmicas (Valente et al. 2008).

\section{CONCLUSÃO}

A espécie Chelonia mydas possui implicações morfológicas do coração, bem como aortas e artérias pulmonares, que a permitem sobreviver a variações de pressão durante o mergulho em apneia. Em virtude do alto grau de relevância desse trabalho no âmbito da morfologia, fisiologia e sistemática filogenética é necessário ampliar os estudos e inter-relacionar com outras espécies de tartarugas em hábitat diversificados para se estabelecer as adaptações com maior precisão.

Agradecimentos.- À equipe de técnicas do Laboratório de Histologia da UFRN pelo auxílio na confecção das lâminas. Ao Aquário Natal por fornecer os animais para a realização deste trabalho e a parceria com o TAMAR-RN fundamental para a concretização deste trabalho.

\section{REFERÊNCIAS}

Ashley L.M. 1955. Laboratory Anatomy of the Turtle. WMC Brow, Dubuque. 110 p.

Best P.V. \& Heath D. 1961. Interpretation of the appearances of the small pulmonary blood vessels in animals. Circ. Res. 9:288-294.

Bisaillon A., Martineau D. \& St-Pierre M.A. 1987. Anatomy of the heart of the beluga whale (Delphinapterus leucas). J. Morphol. 191:89-100.

Bolten A.B. 2000. Técnicas para la medición de tortugas marinas, p.126131. In: Eckert K.L., Bjorndal K.A., Abreu-Grobois F.A. \& Donnelly M. (Eds), Técnicas de Investigación y Manejo para la Conservación de las Tortugas Marinas. Publicación n.4 UICN/CSE Grupo Especialista de Tortugas Marinas. Washington, DC.

Davis D.D. 1964. Anatomy of the heart in bears (Ursidae), and factors influencing the form of the Mammalian heart. J. Morphol. 106:553-568.

Drabek C.M. \& Burns J.M. 2002. Heart and aorta morphology of the deepdiving hooded seal (Cystophora cristata). Can. J. Zoology 80:2030-2036.

Franklin C. \& Axelsson M. 1994. The intrinsic properties of an in situ perfused crocodile heart. J. Exp. Biol. 186:269-288.

Garri R.G. 2006. Comportamento de mergulho do boto-cinza, Sotalia guianensis, na enseada do curral, praia de Pipa-RN, Brasil. Possíveis adaptações cardíacas ao mergulho. Dissertação de Mestrado em Psicobiologia, Universidade Federal do Rio Grande do Norte, Natal, RN. 116p.

Hay G.C., Adams C.R., Broderick A.C., Godley B.J., Lucas D.J., Mercalfe J.D. \& Prior A.A. 2000. The diving behavior of green turtles at Ascension Island. Anim. Behav. 59:577-586.

Hirth H.F. 1997. Synopsis of the Biological data on the Green Turtle Chelonia mydas (Linnaeus, 1758). Biological Report 97, Fish and Wildlife Service, Washington, DC, p.1. 
International Union for Conservation of Nature 2009. Wildlife in a Changing World: an analysis of the 2008 Red List. 180p.

Junin M. \& Belerenian G. 1990. Análisis electrocardiográfico en crías de Otaria flavescens, en relación al desarrollo de la capacidad de buceo profundo y su comparación con el adulto. Anais $4^{\underline{a}}$ Reunión de Trabajo de Especialistas em Mamíferos Acuáticos de America del Sur, Valdivia, Chile, p.35. (Resumo) (Apud Garri 2006)

Junqueira L.C. \& Carneiro J. 2008. Histologia Básica. 11aㅡ ed. Guanabara Koogan, Rio de Janeiro. 524p.

Kierszenbaum A. 2008. Histologia e Biologia Celular: uma introdução à patologia. $2^{\mathrm{a}}$ ed. Elsevier, Rio de Janeiro. 677p.

López-Mendilaharsu M., Rocha C.F.D., Domingo A., Wallace B.P. \& Miller P. 2008. Prolonged, deep dives by the leatherback turtle. JMBA2 - Biodiversity Records. Publicação on-line.

Lutcavage M.E., Bushnell P.G. \& Jones D.R. 1992. Oxygen stores and aerobic metabolism in the Leatherback sea turtle. Can. J. Zoology 70:348-351.

Maia V. 1979. Técnica Histológica. 2ª ed. Atheneu, São Paulo, p.70-136.

Murray M.J. 2006. Cardiopulmonary anatomy and physiology, p.124-134. In: Mader D.R. (Ed.), Reptile Medicine and Surgery. Elsevier Inc., Philadelphia.

Meylan A.B. \& Meylan P.A. 2000 Introducción a la evolución, historias de vida y biología de las tortugas marinas, p.3-5. In: Eckert K.L., Bjorndal K.A., Abreu-Grobois F.A. \& Donnelly M. (Eds), Técnicas de Investigación y Manejo para la Conservación de las Tortugas Marinas. IUCN/SSC Publicación.

Quesada R. \& Madriz L.F. 1986. Coronary vascularization of the marine turtle Chelonia mydas. Revta Biol. Trop. 34:253-258.
Rice M.R. \& Balazs G.H. 2008. Diving behavior of the Hawaiian green turtle (Chelonia mydas) during oceanic migrations. J. Exp. Mar. Biol. Ecol. 356:121-127.

Silva M.R. \& Rodrigues F.P. 2009. Flutuação em tartaruga marinha da espécie Caretta caretta decorrente de pneumonia bacteriana. PubVet, Londrina, 3:11p.

Solomon V., Vijaya M.N. \& Raveen R. 1999. Evolution of the ventricle. Tex. Heart J. 26:168-176.

Southwood A.L., Andrews R.D., Lutcavage M.E.L., Paladino F.V., West N.H., George R.H. \& Jones D.R. 1999. Heart rates and diving behavior of leatherback sea turtles in eastern Pacific Ocean. J. Exp. Biol. 202:11151125.

Santos M.R.D., Ferreira L.S., Batistote C., Grossman A. \& Bellini C. 2009. Valores hematológicos de tartarugas marinhas Chelonia mydas (Linaeus, 1758) juvenis selvagens do Arquipélago de Fernando de Noronha, Pernambuco, Brasil. Braz. J. Vet. Res. Anim. Sci. 46:491-499.

Valete A.L., Parga M.L., Espada Y., Lavin S., Alegre F., Marco I. \& Cuenca R. 2008. Evaluation of Doppler ultrasonography for the measurement of blood flow in young loggerhead sea turtles (Caretta caretta). Vet. J. 176:385-392.

Work T.M. 2000. Manual de Necropsia de Tortugas Marinas para Biologos en Refugios o Areas Remotas. National Wildlife Health Center, Hawaii Field Station. 25p.

Wyneken J. 2001. The Anatomy of Sea Turtle. U.S Techinical Memorandum NMFS-SEFS, Department of Commerce NOAA, Washington. DC. 172p.

Wyneken J. 2009. Normal reptile heart morphology and fuction. Vet. Clin. Exot. Anim. 12:51-63. 